УДК 517.9

\title{
On the Solvability of a System of Two Multidimensional Loaded Parabolic Equations with the Cauchy Data
}

\author{
Galina V. Romanenko* \\ Igor V. Frolenkov ${ }^{\dagger}$ \\ Institute of Mathematics and Computer Science \\ Siberian Federal University \\ Svobodny, 79, Krasnoyarsk, 660041
}

Russia

Received 17.01.2016, received in revised form 03.04.2016, accepted 15.06.2016

We study a multidimensional system of two loaded parabolic equations of a special kind with the Cauchy data. Sufficient conditions for the existence of a solution in the class of smooth bounded functions are obtained. The splitting method at differential level (the method of weak approximation) is used in the proof.

Keywords: inverse problem, direct problem, loaded equation, parabolic equation, weak approximation method, system of partial differential equations, Cauchy problem.

DOI: 10.17516/1997-1397-2016-9-3-364-373.

\section{Introduction}

A study of inverse problems for systems of parabolic equations can be a time-consuming process, therefore, in [1] there was proposed an algorithm to the study of one-dimensional direct problems for systems of parabolic equations, to which inverse problems for loaded systems of a special kind can be reduced. Increasing dimension of a problem leads to estimations obtained in the process become more complex, which significantly slows down the study.

The article presents a generalization of the algorithm proposed in [1] to the multidimensional case. In the present article we propose and investigate the following model: a system of two multidimensional loaded parabolic equations connected by the lower terms with the Cauchy data. Our motivation is that inverse problems for systems of linear or semilinear parabolic equations with unknown coefficients depending only on time variable can be reduced to such a model. The obtained result can be used as a sufficient condition for existence of a solution to auxiliary direct problems. To prove existence of a solution we use the weak approximation method, which is the splitting method at the differential level $[2,3]$.

Another method of the study of systems of multidimensional parabolic equations in a special form is presented in [4]. Similar algorithms for the study of inverse problems for parabolic equations have been previously presented in $[5,6]$. The Cauchy problem for a loaded Burgerstype system has been investigated in [7].

\footnotetext{
*galina.romanencko@yandex.ru

†igor@frolenkov.ru

(c) Siberian Federal University. All rights reserved
} 


\section{Statement of the problem}

In the space $E_{n}$ of variables $x_{1}, \ldots, x_{n}$ choose $r_{i}$ different points $\alpha_{k_{i}}^{i},\left(k_{i}=\overline{1, r_{i}}\right)$ for each variable $x_{i}(i=\overline{1, n})$. In the strip

$$
G_{[0, T]}=\left\{(t, x) \mid 0 \leqslant t \leqslant T, x=\left(x_{1}, \ldots, x_{n}\right) \in E_{n}\right\}
$$

consider the Cauchy problem for the system of loaded nonclassical parabolic equations

$$
\begin{gathered}
u_{t}(t, x)=\sum_{i=1}^{n} a_{1}^{i}\left(t, \bar{\varphi}_{u}(t), \bar{\varphi}_{v}(t)\right) u_{x_{i} x_{i}}+\sum_{i=1}^{n} b_{1}^{i}\left(t, \bar{\varphi}_{u}(t), \bar{\varphi}_{v}(t)\right) u_{x_{i}}+f_{1}\left(t, x, u, v, \bar{\varphi}_{u}(t), \bar{\varphi}_{v}(t)\right), \\
v_{t}(t, x)=\sum_{i=1}^{n} a_{2}^{i}\left(t, \bar{\varphi}_{u}(t), \bar{\varphi}_{v}(t)\right) v_{x_{i} x_{i}}+\sum_{i=1}^{n} b_{2}^{i}\left(t, \bar{\varphi}_{u}(t), \bar{\varphi}_{v}(t)\right) v_{x_{i}}+f_{2}\left(t, x, u, v, \bar{\varphi}_{u}(t), \bar{\varphi}_{v}(t)\right) \\
u(0, x)=u_{0}(x), v(0, x)=v_{0}(x), \quad x \in E_{n},
\end{gathered}
$$

here

$$
\begin{gathered}
\bar{\varphi}_{u}(t)=\left(u\left(t, \alpha_{k_{1}}^{1}, \ldots, \alpha_{k_{n}}^{n}\right), \quad D_{x}^{s} u\left(t, \alpha_{k_{1}}^{1}, \ldots, \alpha_{k_{n}}^{n}\right)\right), \\
\bar{\varphi}_{v}(t)=\left(v\left(t, \alpha_{k_{1}}^{1}, \ldots, \alpha_{k_{n}}^{n}\right), \quad D_{x}^{s} v\left(t, \alpha_{k_{1}}^{1}, \ldots, \alpha_{k_{n}}^{n}\right)\right), \\
s=\left(s_{1}, \ldots, s_{n}\right), s_{i}=0,1, \ldots, \widetilde{p}_{i} ; \quad k_{i}=1, \ldots, r_{i} ; i=1, \ldots, n ; \\
D_{x}^{s} \xi(x)=D_{\left(x_{1}, x_{2}, \ldots, x_{n}\right)}^{\left(s_{1}, s_{2}, \ldots, s_{n}\right)} \xi(x)=\frac{\partial^{|s|} \xi\left(x_{1}, x_{2}, \ldots, x_{n}\right)}{\partial x_{1}^{s_{1}} \partial x_{2}^{s_{2}} \ldots \partial x_{n}^{s_{n}}},
\end{gathered}
$$

where $s=\left(s_{1}, s_{2}, \ldots, s_{n}\right)$ is a multiindex, $s_{r} \geqslant 0$ is integer, $r=1,2, \ldots, n,|s|=s_{1}+\ldots+s_{n}$.

Henceforth, choose and fix the constants $p_{i}=\max \left\{\widetilde{p}_{i}, 2\right\}, i=1, \ldots, n$. Also, introduce the following definitions.

Definition 1. Denote by $Z_{x_{1}, \ldots, x_{n}}^{p_{1}, \ldots, p_{n}}\left(\left[0, t^{*}\right]\right)$ the set of the functions $u(t, x), v(t, x)$ defined in $G_{\left[0, t^{*}\right]}$ belonging to the class $C_{t, x_{1}, \ldots, x_{n}}^{1, p_{1}, \ldots, x_{n}}\left(G_{\left[0, t^{*}\right]}\right)$ where

$$
\begin{aligned}
C_{t, x_{1}, \ldots, x_{n}}^{1, p_{1}, \ldots, p_{n}}\left(G_{\left[0, t^{*}\right]}\right)=\left\{\psi(t, x) \mid \frac{\partial \psi}{\partial t}, D_{x}^{s} \psi\right. & \in C\left(G_{\left[0, t^{*}\right]}\right), \\
& \left.s=\left(s_{1}, \ldots, s_{n}\right), s_{i}=0,1, \ldots, p_{i}, i=1, \ldots, n\right\},
\end{aligned}
$$

and bounded in $G_{\left[0, t^{*}\right]}$ together with their derivatives occurring in (1),

$$
\sum_{\substack{s=\left(s_{1}, \ldots, s_{n}\right), s_{i}=0,1, \ldots, p_{i} \\ i=1, \ldots, n}}\left(\left|D_{x}^{s} u(t, x)\right|+\left|D_{x}^{s} v(t, x)\right|\right) \leqslant C .
$$

Definition 2. By a classical solution to problem (1), (2) in $G_{\left[0, t^{*}\right]}$ we mean a pair of functions $u(t, x), v(t, x) \in Z_{x_{1}, \ldots, x_{n}}^{p_{1}, \ldots, p_{n}}\left(\left[0, t^{*}\right]\right)$, satisfying (1), (2) in $G_{\left[0, t^{*}\right]}$.

Here $0<t^{*} \leqslant T$ is a fixed constant. If $t^{*}$ depends on the constants bounding the initial data and $t^{*} \leqslant T$ then we say that the functions $u(t, x), v(t, x)$ are a solution (1), (2) 'in the small'. If $t^{*}$ is fixed and $t^{*}=T$ for every collection of initial data satisfying the sufficient solvability conditions then we say that $u(t, x), v(t, x)$ are a solution (1), (2) 'in the whole' (or use the term global solvability). 


\section{The main result}

Suppose that the following conditions hold:

Condition 1. The functions $a_{1}^{i}, a_{2}^{i}, b_{1}^{i}, b_{2}^{i}(i=\overline{1, n})$ are real-valued, defined, and continuous for all values of their arguments; $a_{1}^{i}, a_{2}^{i}$ satisfy the conditions $a_{1}^{i} \geqslant a_{0}>0, a_{2}^{i} \geqslant a_{0}>0, a_{0}$ is a constant, $(i=\overline{1, n})$. For all $t_{1} \in(0, T], q(t, x), w(t, x) \in Z_{x_{1}, \ldots, x_{n}}^{p_{1}+2, \ldots, p_{n}+2}\left(\left[0, t_{1}\right]\right)$ the relation holds

$$
\begin{aligned}
\sum_{i=1}^{n}\left(\left|a_{1}^{i}\left(t, \bar{\varphi}_{q}(t), \bar{\varphi}_{w}(t)\right)\right|\right. & +\left|a_{2}^{i}\left(t, \bar{\varphi}_{q}(t), \bar{\varphi}_{w}(t)\right)\right|+ \\
& \left.+\left|b_{1}^{i}\left(t, \bar{\varphi}_{q}(t), \bar{\varphi}_{w}(t)\right)\right|+\left|b_{2}^{i}\left(t, \bar{\varphi}_{q}(t), \bar{\varphi}_{w}(t)\right)\right|\right) \leqslant P_{\gamma_{1}}\left(S_{q, w}(t)\right) .
\end{aligned}
$$

Remark 1. In Conditions 1 and 3 , by $\gamma_{1}, \gamma_{2} \geqslant 0$ we mean some fixed real numbers,

$$
\begin{aligned}
& S_{q, w}(t)=\sum_{\substack{s=\left(s_{1}, \ldots, s_{n}\right), s_{i}=0,1, \ldots, p_{i}+2, i=1, \ldots, n}}\left(\sup _{0<\xi \leqslant t} \sup _{x \in E_{n}}\left|D_{x}^{s} q(\xi, x)\right|+\sup _{0<\xi \leqslant t} \sup _{x \in E_{n}}\left|D_{x}^{s} w(\xi, x)\right|\right), \\
& q(t, x), w(t, x) \in Z_{x_{1}, \ldots, x_{n}}^{p_{1}+2, \ldots, p_{n}+2}\left(\left[0, t_{1}\right]\right),
\end{aligned}
$$

$P_{\zeta}(y)=\tilde{C}\left(1+y+\cdots+y^{\zeta}\right), \tilde{C}>1$ is a constant independent of $q(t, x), w(t, x)$ and their derivatives.

Condition 2. The functions $u_{0}(x), v_{0}(x)$ are real-valued and have all continuous derivatives occurring in the following relation and satisfying it:

$$
\sum_{\substack{s=\left(s_{1}, \ldots, s_{n}\right) \\ s_{i}=0,1, \ldots, p_{i}+2, i=1, \ldots, n}}\left(\left|D_{x}^{s} u_{0}(x)\right|+\left|D_{x}^{s} v_{0}(x)\right|\right) \leqslant C .
$$

Condition 3. The functions $f_{1}, f_{2}$ are real-valued, defined, and continuous for all values of their arguments. For all $t_{1} \in(0, T], q(t, x), w(t, x) \in Z_{x_{1}, \ldots, x_{n}}^{p_{1}+2, \ldots, p_{n}+2}\left(\left[0, t_{1}\right]\right)$ as functions of the variables $(t, x) \in G_{\left[0, t_{1}\right]}$, these functions are continuous and have continuous derivatives occurring in the relation

$$
\begin{aligned}
& \sum_{\substack{s=\left(s_{1}, \ldots, s_{n}\right), s_{i}=0, \ldots, \ldots, p_{i}+2, i=1, \ldots, n}}\left(\left|D_{x}^{s} f_{1}\left(t, x, q, w, \bar{\varphi}_{q}(t), \bar{\varphi}_{w}(t)\right)\right|+\right. \\
& \left.+\left|D_{x}^{s} f_{2}\left(t, x, q, w, \bar{\varphi}_{q}(t), \bar{\varphi}_{w}(t)\right)\right|\right) \leqslant P_{\gamma_{2}}\left(S_{q, w}(t)\right) .
\end{aligned}
$$

Theorem. Suppose that Conditions 1-3 hold. Then

[a] If Conditions 1-3 hold for $\gamma_{1} \geqslant 0, \gamma_{2}=0$ or $\gamma_{2}=1$, then a classical solution $\{u(t, x), v(t, x)\}$ to problem (1), (2) exists in the class $Z_{x_{1}, \ldots, x_{n}}^{p_{1}, \ldots, p_{n}}([0, T])$.

[b] If Conditions 1-3 hold for $\gamma_{1} \geqslant 0, \gamma_{2}>1$, then there exists a constant $t^{*}, 0<t^{*} \leqslant T$, depending on the constant $\tilde{C}$ of (5), (6), such that a classical solution $\{u(t, x), v(t, x)\}$ to problem (1), (2) exists in the class $Z_{x_{1}, \ldots, x_{n}}^{p_{1}, \ldots, p_{n}}\left(\left[0, t^{*}\right]\right)$. 
Proof. The proof is conducted by using the splitting method on the differential level. We use the spitting of the initial problem into two fractional steps with time shift by $\left(t-\frac{\tau}{2}\right)$ in the traces of the unknown functions and nonlinear terms:

$$
\begin{gathered}
u_{t}^{\tau}(t, x)=2 \sum_{i=1}^{n} a_{1}^{i}\left(t, \bar{\varphi}_{u^{\tau}}^{\tau}\left(t-\frac{\tau}{2}\right), \bar{\varphi}_{v^{\tau}}^{\tau}\left(t-\frac{\tau}{2}\right)\right) u_{x_{i} x_{i}}^{\tau}+2 \sum_{i=1}^{n} b_{1}^{i}\left(t, \bar{\varphi}_{u^{\tau}}^{\tau}\left(t-\frac{\tau}{2}\right), \bar{\varphi}_{v^{\tau}}^{\tau}\left(t-\frac{\tau}{2}\right)\right) u_{x_{i}}^{\tau}, \\
v_{t}^{\tau}(t, x)=2 \sum_{i=1}^{n} a_{2}^{i}\left(t, \bar{\varphi}_{u^{\tau}}^{\tau}\left(t-\frac{\tau}{2}\right), \bar{\varphi}_{v^{\tau}}^{\tau}\left(t-\frac{\tau}{2}\right)\right) v_{x_{i} x_{i}}^{\tau}+2 \sum_{i=1}^{n} b_{2}^{i}\left(t, \bar{\varphi}_{u^{\tau}}^{\tau}\left(t-\frac{\tau}{2}\right), \bar{\varphi}_{v^{\tau}}^{\tau}\left(t-\frac{\tau}{2}\right)\right) v_{x_{i}}^{\tau}, \\
n \tau<t \leqslant\left(n+\frac{1}{2}\right) \tau, \\
u_{t}^{\tau}(t, x)=2 f_{1}\left(t-\frac{\tau}{2}, x, u^{\tau}\left(t-\frac{\tau}{2}, x\right), v^{\tau}\left(t-\frac{\tau}{2}, x\right), \bar{\varphi}_{u^{\tau}}^{\tau}\left(t-\frac{\tau}{2}\right), \bar{\varphi}_{v^{\tau}}^{\tau}\left(t-\frac{\tau}{2}\right)\right), \\
v_{t}^{\tau}(t, x)=2 f_{2}\left(t-\frac{\tau}{2}, x, u^{\tau}\left(t-\frac{\tau}{2}, x\right), v^{\tau}\left(t-\frac{\tau}{2}, x\right), \bar{\varphi}_{u^{\tau}}^{\tau}\left(t-\frac{\tau}{2}\right), \bar{\varphi}_{v^{\tau}}^{\tau}\left(t-\frac{\tau}{2}\right)\right), \\
\left(n+\frac{1}{2}\right) \tau<t \leqslant(n+1) \tau, \\
\left.u^{\tau}(t, x)\right|_{t \leqslant 0}=u_{0}(x),\left.\quad v^{\tau}(t, x)\right|_{t \leqslant 0}=v_{0}(x), \quad x \in E_{n},
\end{gathered}
$$

here $n=0, \ldots, N-1, \quad N \tau=T$,

$$
\begin{array}{r}
\bar{\varphi}_{u^{\tau}}^{\tau}\left(t-\frac{\tau}{2}\right)=\left(u^{\tau}\left(t-\frac{\tau}{2}, \alpha_{k_{1}}^{1}, \ldots, \alpha_{k_{n}}^{n}\right), D_{x}^{s} u^{\tau}\left(t-\frac{\tau}{2}, \alpha_{k_{1}}^{1}, \ldots, \alpha_{k_{n}}^{n}\right)\right), \\
\bar{\varphi}_{v^{\tau}}^{\tau}\left(t-\frac{\tau}{2}\right)=\left(v^{\tau}\left(t-\frac{\tau}{2}, \alpha_{k_{1}}^{1}, \ldots, \alpha_{k_{n}}^{n}\right), D_{x}^{s} v^{\tau}\left(t-\frac{\tau}{2}, \alpha_{k_{1}}^{1}, \ldots, \alpha_{k_{n}}^{n}\right)\right), \\
s=\left(s_{1}, \ldots, s_{n}\right), s_{i}=0,1, \ldots, \widetilde{p}_{i}, k_{i}=1, \ldots, r_{i}, i=1, \ldots, n
\end{array}
$$

Now, establish a priori estimates that guarantee the compactness of the family of solutions $\left\{u^{\tau}(t, x), v^{\tau}(t, x)\right\}$ to $(7),(8)$ in classes $C_{t, x_{1}, \ldots, x_{n}}^{1, p_{1}, \ldots, p_{n}}\left(G_{\left[0, t^{*}\right]}\right)$ for some constant $0<t^{*} \leqslant T$.

Refer as the $n$-th integer time step to the half-interval $(n \tau,(n+1) \tau], n=0,1, \ldots, N-1$.

Introduce the notation:

$$
\begin{aligned}
& S_{u, v}(0)=\sum_{\substack{s=\left(s_{1}, \ldots, s_{n}\right), s_{i}=0,1, \ldots, p_{i}+2, i=1, \ldots, n}}\left(\sup _{x \in E_{n}}\left|D_{x}^{s} u_{0}(x)\right|+\sup _{x \in E_{n}}\left|D_{x}^{s} v_{0}(x)\right|\right), \\
& S_{u^{\tau}, v^{\tau}}(t)=\sum_{\substack{s=\left(s_{1}, \ldots, s_{n}\right), s_{i}=0,1, \ldots, p_{i}+2, i=1, \ldots, n}}\left(\sup _{n \tau<\xi \leqslant t \leqslant(n+1) \tau} \sup _{x \in E_{n}}\left|D_{x}^{s} u^{\tau}(\xi, x)\right|+\sup _{n \tau<\xi \leqslant t \leqslant(n+1) \tau} \sup _{x \in E_{n}}\left|D_{x}^{s} v^{\tau}(\xi, x)\right|\right), \\
& n \tau<t \leqslant(n+1) \tau .
\end{aligned}
$$

The following hold:

1. $\left|D_{x}^{s} u^{\tau}(\xi, x)\right|+\left|D_{x}^{s} v^{\tau}(\xi, x)\right| \leqslant S_{u^{\tau}, v^{\tau}}(t), \quad \xi \in(n \tau, t]$,

$$
t \in(n \tau,(n+1) \tau], \quad s=\left(s_{1}, \ldots, s_{n}\right), s_{i}=0,1, \ldots, p_{i}+2, i=\overline{1, n}
$$

2. the functions $S_{u^{\tau}, v^{\tau}}(t)$ are nonnegative and nondecreasing on each time interval $(n \tau,(n+1) \tau]$. 
[a]. At the first fractional step $t \in\left(0, \frac{\tau}{2}\right]$, for the solution $u^{\tau}, v^{\tau}$ to $(7)$ with initial data (9), by Conditions $1-3$ and the maximum principle, we obtain the estimate

$$
\begin{aligned}
& \left|u^{\tau}(\xi, x)\right| \leqslant \sup _{x \in E_{n}}\left|u_{0}(x)\right|, \\
& \left|v^{\tau}(\xi, x)\right| \leqslant \sup _{x \in E_{n}}\left|v_{0}(x)\right|, \quad 0<\xi \leqslant t, \quad 0<t \leqslant \frac{\tau}{2} .
\end{aligned}
$$

Summing up the obtained estimates, we have

$$
\left|u^{\tau}(\xi, x)\right|+\left|v^{\tau}(\xi, x)\right| \leqslant \sup _{x \in E_{n}}\left|u_{0}(x)\right|+\sup _{x \in E_{n}}\left|v_{0}(x)\right|, 0<\xi \leqslant t, \quad 0<t \leqslant \frac{\tau}{2} .
$$

We can apply the operation of differentiation $D_{x}^{s}$ to problem $(7),(9) s=\left(s_{1}, \ldots, s_{n}\right)$, $s_{i}=0,1, \ldots, p_{i}+2, i=1, \ldots, n$. By Condition 1,2 , obtain:

$$
\begin{gathered}
\left|D_{x}^{s} u^{\tau}(\xi, x)\right| \leqslant \sup _{x \in E_{n}}\left|D_{x}^{s} u_{0}(x)\right|, \\
\left|D_{x}^{s} v^{\tau}(\xi, x)\right| \leqslant \sup _{x \in E_{n}}\left|D_{x}^{s} v_{0}(x)\right|, \\
0<\xi \leqslant t, t \in\left(0, \frac{\tau}{2}\right], s=\left(s_{1}, \ldots, s_{n}\right), s_{i}=0,1, \ldots, p_{i}+2, i=1, \ldots, n .
\end{gathered}
$$

Apply $\sup _{x \in E_{n}}$ to both sides of the inequalities (12), (13), then $\sup _{0<\xi \leqslant t}$, and sum the results up, taking into account the notation (10), we get

$$
S_{u^{\tau}, v^{\tau}}(t) \leqslant S_{u, v}(0), \quad 0<t \leqslant \frac{\tau}{2}
$$

Consider the second fractional step of the zero integer step $t \in\left(\frac{\tau}{2}, \tau\right]$. Integrating the system of equations (8) with respect to the time variable over the interval $\left(\frac{\tau}{2}, \xi\right], \xi \in\left(\frac{\tau}{2}, t\right]$ and $\frac{\tau}{2}<t \leqslant \tau$, we obtain

$$
\begin{aligned}
& u^{\tau}(\xi, x)=u^{\tau}\left(\frac{\tau}{2}, x\right)+2 \int_{\frac{\tau}{2}}^{\xi} f_{1}\left(\theta-\frac{\tau}{2}, x, u^{\tau}\left(\theta-\frac{\tau}{2}, x\right), v^{\tau}\left(\theta-\frac{\tau}{2}, x\right), \bar{\varphi}_{u^{\tau}}^{\tau}\left(\theta-\frac{\tau}{2}\right), \bar{\varphi}_{v^{\tau}}^{\tau}\left(\theta-\frac{\tau}{2}\right)\right) d \theta, \\
& v^{\tau}(\xi, x)=v^{\tau}\left(\frac{\tau}{2}, x\right)+2 \int_{\frac{\tau}{2}}^{\xi} f_{2}\left(\theta-\frac{\tau}{2}, x, u^{\tau}\left(\theta-\frac{\tau}{2}, x\right), v^{\tau}\left(\theta-\frac{\tau}{2}, x\right), \bar{\varphi}_{u^{\tau}}^{\tau}\left(\theta-\frac{\tau}{2}\right), \bar{\varphi}_{v^{\tau}}^{\tau}\left(\theta-\frac{\tau}{2}\right)\right) d \theta, \\
& \xi \in\left(\frac{\tau}{2}, t\right], \frac{\tau}{2}<t \leqslant \tau, \\
& \left|u^{\tau}(\xi, x)\right| \leqslant\left|u^{\tau}\left(\frac{\tau}{2}, x\right)\right|+ \\
& +2 \int_{\frac{\tau}{2}}^{\xi}\left|f_{1}\left(\theta-\frac{\tau}{2}, x, u^{\tau}\left(\theta-\frac{\tau}{2}, x\right), v^{\tau}\left(\theta-\frac{\tau}{2}, x\right), \bar{\varphi}_{u^{\tau}}^{\tau}\left(\theta-\frac{\tau}{2}\right), \bar{\varphi}_{v^{\tau}}^{\tau}\left(\theta-\frac{\tau}{2}\right)\right)\right| d \theta, \\
& \left|v^{\tau}(\xi, x)\right| \leqslant\left|v^{\tau}\left(\frac{\tau}{2}, x\right)\right|+ \\
& +2 \int_{\frac{\tau}{2}}^{\xi}\left|f_{2}\left(\theta-\frac{\tau}{2}, x, u^{\tau}\left(\theta-\frac{\tau}{2}, x\right), v^{\tau}\left(\theta-\frac{\tau}{2}, x\right), \bar{\varphi}_{u^{\tau}}^{\tau}\left(\theta-\frac{\tau}{2}\right), \bar{\varphi}_{v^{\tau}}^{\tau}\left(\theta-\frac{\tau}{2}\right)\right)\right| d \theta, \\
& \xi \in\left(\frac{\tau}{2}, t\right], \frac{\tau}{2}<t \leqslant \tau,
\end{aligned}
$$

The last inequality, Condition 3 (inequality (6)) and the conditions of case [a] imply

$$
\begin{aligned}
& \left|u^{\tau}(\xi, x)\right| \leqslant\left|u^{\tau}\left(\frac{\tau}{2}, x\right)\right|+2 \int_{\frac{\tau}{2}}^{\tau} \tilde{C}\left(1+S_{u^{\tau}, v^{\tau}}\left(\theta-\frac{\tau}{2}\right)\right), d \theta, \quad \xi \in\left(\frac{\tau}{2}, t\right], \frac{\tau}{2}<t \leqslant \tau, \\
& \left|v^{\tau}(\xi, x)\right| \leqslant\left|v^{\tau}\left(\frac{\tau}{2}, x\right)\right|+2 \int_{\frac{\tau}{2}}^{\tau} \tilde{C}\left(1+S_{u^{\tau}, v^{\tau}}\left(\theta-\frac{\tau}{2}\right)\right), d \theta, \quad
\end{aligned}
$$


whence, using the properties $S_{u^{\tau}, v^{\tau}}(t)$ in (10) and summing up the inequalities, we infer

$$
\begin{aligned}
\left|u^{\tau}(\xi, x)\right|+\left|v^{\tau}(\xi, x)\right| \leqslant\left|u^{\tau}\left(\frac{\tau}{2}, x\right)\right|+\left|v^{\tau}\left(\frac{\tau}{2}, x\right)\right|+\tilde{C}\left(1+S_{u^{\tau}, v^{\tau}}\left(\frac{\tau}{2}\right)\right) \tau & \\
\xi & \in\left(\frac{\tau}{2}, t\right], \frac{\tau}{2}<t \leqslant \tau .
\end{aligned}
$$

We apply the operation of of differentiation $D_{x}^{s}, s=\left(s_{1}, \ldots, s_{n}\right), s_{i}=0,1, \ldots, p_{i}+2, i=$ $1, \ldots, n$, to the equation (8) and then integrate with respect to time variable. By condition 3 , we obtain

$$
\begin{aligned}
\left|D_{x}^{s} u^{\tau}(\xi, x)\right|+\left|D_{x}^{s} v^{\tau}(\xi, x)\right| & \leqslant\left|D_{x}^{s} u^{\tau}\left(\frac{\tau}{2}, x\right)\right|+\left|D_{x}^{s} v^{\tau}\left(\frac{\tau}{2}, x\right)\right|+\tilde{C}\left(1+S_{u^{\tau}, v^{\tau}}^{\tau}\left(\frac{\tau}{2}\right)\right) \tau \\
\xi & \in\left(\frac{\tau}{2}, t\right], \frac{\tau}{2}<t \leqslant \tau, \quad s=\left(s_{1}, \ldots, s_{n}\right), s_{i}=0,1, \ldots, p_{i}+2, i=1, \ldots, n .
\end{aligned}
$$

Apply $\sup _{x \in E_{n}}$ and $\sup _{0<\xi \leqslant t}$ to both sides of the inequalities (15), (16) and sum them. By (10), we see that

$$
S_{u^{\tau}, v^{\tau}}(t) \leqslant S_{u^{\tau}, v^{\tau}}\left(\frac{\tau}{2}\right)+\tilde{C}\left(1+S_{u^{\tau}, v^{\tau}}\left(\frac{\tau}{2}\right)\right) \tau, \frac{\tau}{2}<t \leqslant \tau .
$$

Taking into account (14), from this inequality at the zero integer time step we obtain:

$$
S_{u^{\tau}, v^{\tau}}(t) \leqslant S_{u, v}(0)+C\left(1+S_{u, v}(0)\right) \tau, 0<t \leqslant \tau,
$$

here and below we assume that $C>1$ are some (generally speaking, different) constants bounding the input data in Conditions 1-3 and independent of the splitting parameter $\tau$.

$$
S_{u^{\tau}, v^{\tau}}(t) \leqslant S_{u, v}(0)+1-1+C\left(1+S_{u, v}(0)\right) \tau \leqslant\left(1+S_{u, v}(0)\right)(1+C \tau)-1 \leqslant\left(1+S_{u, v}(0)\right) e^{C \tau}-1 .
$$

At the first integer time step $t \in(\tau, 2 \tau]$, arguing similarly to the zero integer step, we have

$$
S_{u^{\tau}, v^{\tau}}(t) \leqslant\left(1+S_{u^{\tau}, v^{\tau}}(\tau)\right) e^{C \tau}-1 \leqslant\left(1+S_{u, v}(0)\right) e^{2 C \tau}-1 .
$$

In finitely many steps, on the interval $((N-1) \tau, N \tau]$ we get

$$
S_{u^{\tau}, v^{\tau}}(t) \leqslant\left(1+S_{u, v}(0)\right) e^{N C \tau}-1=\left(1+S_{u, v}(0)\right) \cdot e^{C T}-1 \leqslant C, \forall t \in((N-1) \tau, N \tau] .
$$

As a result, on $[0, T]$ we obtain

$$
S_{u^{\tau}, v^{\tau}}(t) \leqslant\left(1+S_{u, v}(0)\right) \cdot e^{C T}-1 \leqslant C, \forall t \in[0, T] .
$$

Thus we have proved the following estimates, which are uniform in $\tau$ :

$$
\begin{aligned}
& \left|D_{x}^{s} u^{\tau}(t, x)\right|+\left|D_{x}^{s} v^{\tau}(t, x)\right| \leqslant C, \\
& \quad s=\left(s_{1}, \ldots, s_{n}\right), s_{i}=0,1, \ldots, p_{i}+2, i=1, \ldots, n, \quad(t, x) \in G_{[0, T]} .
\end{aligned}
$$

Estimates (18) imply that the right-hand sides of (7), (8) are bounded uniformly in $\tau$ on each time step, and hence the left-hand sides of the equations are bounded uniformly in $\tau$ :

$$
\left|u_{t}^{\tau}(t, x)\right|+\left|v_{t}^{\tau}(t, x)\right| \leqslant C,(t, x, z) \in G_{[0, T]} .
$$

We apply operation of differentiation $D_{x}^{s}$ for $s=\left(s_{1}, \ldots, s_{n}\right), s_{i}=0,1, \ldots, p_{i}$ to $(7),(8)$, by (18), we obtain

$$
\left|D_{x}^{s} u_{t}^{\tau}(t, x)\right|+\left|D_{x}^{s} v_{t}^{\tau}(t, x)\right| \leqslant C, s=\left(s_{1}, \ldots, s_{n}\right), s_{i}=0,1, \ldots, p_{i}, i=1, \ldots, n .
$$


Put $G_{[0, T]}^{M}=\left\{(t, x) \mid 0 \leqslant t \leqslant T, x=\left(x_{1}, \ldots, x_{n}\right)<M\right\}$. Estimates (18), (19) guarantee the fulfillment of the conditions of the Arzela Compactness Theorem. By this theorem, some subsequences $\left\{u^{\tau_{k}}(t, x), v^{\tau_{k}}(t, x)\right\}$ of the sequences $\left\{u^{\tau}(t, x), v^{\tau}(t, x)\right\}$ of solutions to (7), (9) converge together with all relevant derivatives to the functions $u(t, x) \in C_{t, x_{1}, \ldots, x_{n}}^{0, p_{1}, \ldots, p_{n}}\left(G_{[0, T]}\right)$, $v(t, x) \in C_{t, x_{1}, \ldots, x_{n}}^{0, p_{1}, \ldots, p_{n}}\left(G_{[0, T]}\right)$ respectively, which, by the theorem of convergence of the weak approximation method, are the solution to (1), (2) (by virtue of the arbitrariness of M); moreover, $u(t, x) \in C_{t, x_{1}, \ldots, x_{n}}^{1, p_{1}, \ldots, p_{n}}\left(G_{[0, T]}\right), v(t, x) \in C_{t, x_{1}, \ldots, x_{n}}^{1, p_{1}, \ldots, p_{n}}\left(G_{[0, T]}\right)$. Case [a] is proved.

For case $[\mathrm{b}]$, repeating analogous arguments at the first fractional step, we obtain some estimate similar to (14).

At the second fractional step, by the hypotheses of the theorem, we infer the estimate

$$
\begin{aligned}
& \left|D_{x}^{s} u^{\tau}(\xi, x)\right|+\left|D_{x}^{s} v^{\tau}(\xi, x)\right| \leqslant\left|D_{x}^{s} u^{\tau}\left(\frac{\tau}{2}, x\right)\right|+\left|D_{x}^{s} v^{\tau}\left(\frac{\tau}{2}, x\right)\right|+ \\
& \quad+2 \int_{\frac{\tau}{2}}^{t} P_{\gamma_{2}}\left(S_{u^{\tau}, v^{\tau}}\left(\theta-\frac{\tau}{2}\right)\right) d \theta, \xi \in\left(\frac{\tau}{2}, t\right], \frac{\tau}{2}<t \leqslant \tau, \quad j=1, \ldots, p+2 .
\end{aligned}
$$

Summing the last inequality and using the notation (10) at the second fractional step, we obtain

$$
S_{u^{\tau}, v^{\tau}}(t) \leqslant S_{u^{\tau}, v^{\tau}}\left(\frac{\tau}{2}\right)+C \int_{\frac{\tau}{2}}^{t} P_{\gamma_{2}}\left(S_{u^{\tau}, v^{\tau}}\left(\theta-\frac{\tau}{2}\right)\right) d \theta, \frac{\tau}{2}<t \leqslant \tau,
$$

which, since $S_{u^{\tau}, v^{\tau}}$ is nondecreasing in $\left(\frac{\tau}{2}, \tau\right]$, implies

$$
S_{u^{\tau}, v^{\tau}}(t) \leqslant S_{u^{\tau}, v^{\tau}}\left(\frac{\tau}{2}\right)+C \int_{\frac{\tau}{2}}^{t} P_{\gamma_{2}}\left(S_{u^{\tau}, v^{\tau}}(\theta)\right) d \theta, \frac{\tau}{2}<t \leqslant \tau,
$$

Consider the Cauchy problem for the ordinary differential equation

$$
\frac{d \omega(t)}{d t}=C P_{\gamma_{2}}(\omega(t)), \quad \omega(0)=S_{u, v}(0)
$$

Recall that $P_{\gamma_{2}}(y)=\tilde{C}\left(1+y+\cdots+y^{\gamma_{2}}\right), \gamma_{2}>1$ is an integer constant, and the constants $C, \tilde{C}$ are independent of the splitting parameter $\tau$. By the Cauchy theorem, there is a constant $0<t^{*} \leqslant T$ such that a solution to this problem $\omega \in C^{1}\left[0, t^{*}\right]$, where $t^{*}$ depends on $C, \tilde{C}$ and initial condition $S_{u, v}(0)$. Obviously, $\omega(t)$ is a strictly increasing function. We have

Lemma 1. It follows from (20), (21) that if $S_{u^{\tau}, v^{\tau}}\left(t_{0}\right) \leqslant \omega\left(t_{0}\right)$ for some $t_{0} \in\left(0, t^{*}\right)$, then $S_{u^{\tau}, v^{\tau}}(t) \leqslant \omega(t), t \in\left[t_{0}, t^{*}\right]$.

The proof of lemma is in [1].

Thus, we have proved the following estimates uniform over $\tau$ :

$$
\begin{aligned}
& \left|D_{x}^{s} u^{\tau}(t, x)\right|+\left|D_{x}^{s} v^{\tau}(t, x)\right| \leqslant C, \\
& \quad s=\left(s_{1}, \ldots, s_{n}\right), s_{i}=0,1, \ldots, p_{i}+2, i=1, \ldots, n, \quad(t, x) \in G_{\left[0, t^{*}\right]} .
\end{aligned}
$$

Repeating the arguments similar to the arguments in $[\mathrm{a}]$, we infer that some subsequences $\left\{u^{\tau_{k}}(t, x), v^{\tau_{k}}(t, x)\right\}$ of the sequences $\left\{u^{\tau}(t, x), v^{\tau}(t, x)\right\}$ of solutions to (7)-(9) converge together with all relevant derivatives to the functions $u(t, x) \in C_{t, x_{1}, \ldots, x_{n}}^{0, p_{n}, \ldots, p_{n}}\left(G_{\left[0, t^{*}\right]}\right)$, $v(t, x) \in C_{t, x_{1}, \ldots, x_{n}}^{0, p_{1}, \ldots, p_{n}}\left(G_{\left[0, t^{*}\right]}\right)$ respectively, which, by the theorem of the convergence of the weak approximation method, are a solution to (1), (2); moreover, $u(t, x) \in C_{t, x_{1}, \ldots, x_{n}}^{1, p_{1}, \ldots, p_{n}}\left(G_{\left[0, t^{*}\right]}\right)$, $v(t, x) \in C_{t, x_{1}, \ldots, x_{n}}^{1, p_{1}, \ldots, p_{n}}\left(G_{\left[0, t^{*}\right]}\right)$..

Thus, we have proved the existence of a solution "in the small"; i.e., case [b] is proved. The proof of Theorem is complete. 


\section{An example of the application of theorem}

We examine the following Cauchy problem for a system of two-dimensional parabolic equations.

Consider in the domain $G_{[0, T]}=\left\{(t, x) \mid 0 \leqslant t \leqslant T,(x, z) \in E_{2}\right\}$ the system

$$
\begin{gathered}
\left\{\begin{array}{c}
u_{t}(t, x, z)=u_{x x}+u_{z z}+u+v+\lambda(t) F_{1}(t, x, z) \\
v_{t}(t, x, z)=(\cos (t)+3) v_{x x}+v_{z z}+v_{x}+v_{z}+u+v+F_{2}(t, x, z),
\end{array}\right. \\
u(0, x, z)=u_{0}(x, z), \quad v(0, x, z)=v_{0}(x, z), \quad(x, z) \in E_{2} .
\end{gathered}
$$

The function $\lambda(t)$ is to be determined simultaneously with the solution $u(t, x, z), v(t, x, z)$ of the problem.

The overdetermination condition is given by

$$
u(t, \alpha, \beta)=\psi(t)
$$

( $\alpha, \beta$ are constants), the compatibility condition holds

$$
u_{0}(\alpha, \beta)=\psi(0) .
$$

About the input data we assume that they are known functions, sufficiently smooth, have all continuous derivatives occurring in the relations and satisfy them

$$
\begin{gathered}
F_{1}(t, \alpha, \beta) \geqslant \delta>0, \quad \delta \text { is a constant. } \\
|\psi(t)|+\left|\psi^{\prime}(t)\right|+\left|\frac{\partial^{k_{1}+k_{2}} u_{0}}{\partial x^{k_{1}} \partial z^{k_{2}}}\right|+\left|\frac{\partial^{k_{1}+k_{2}} v_{0}}{\partial x^{k_{1}} \partial z^{k_{2}}}\right|+\left|\frac{\partial^{k_{1}+k_{2}} F_{1}}{\partial x^{k_{1}} \partial z^{k_{2}}}\right|+\left|\frac{\partial^{k_{1}+k_{2}} F_{2}}{\partial x^{k_{1}} \partial z^{k_{2}}}\right| \leqslant C, \\
k_{1}, k_{2}=0,1, \ldots, 4 .
\end{gathered}
$$

By using the overdetermination conditions (24), the problem (22), (23) is reduced to the auxiliary direct problem:

$$
\begin{gathered}
\left\{\begin{array}{c}
u_{t}(t, x, z)=u_{x x}+u_{z z}+u+v+\frac{\psi^{\prime}(t)-u_{x x}(t, \alpha, \beta)-u_{z z}(t, \alpha, \beta)-\psi-v(t, \alpha, \beta)}{F_{1}(t, \alpha, \beta)} F_{1}(t, x, z), \\
v_{t}(t, x, z)=(\cos (t)+3) v_{x x}+v_{z z}+v_{x}+v_{z}+u+v+F_{2}(t, x, z),
\end{array}\right. \\
u(0, x, z)=u_{0}(x, z), \quad v(0, x, z)=v_{0}(x, z), \quad(x, z) \in E_{2} .
\end{gathered}
$$

The problem (26), (27) fits into the model proposed above. Here

$$
\begin{gathered}
\begin{array}{r}
a_{1}^{1}\left(t, \bar{\varphi}_{u}(t), \bar{\varphi}_{v}(t)\right)=a_{1}^{2}\left(t, \bar{\varphi}_{u}(t), \bar{\varphi}_{v}(t)\right)=a_{2}^{2}\left(t, \bar{\varphi}_{u}(t), \bar{\varphi}_{v}(t)\right)= \\
=b_{1}^{2}\left(t, \bar{\varphi}_{u}(t), \bar{\varphi}_{v}(t)\right)=b_{2}^{2}\left(t, \bar{\varphi}_{u}(t), \bar{\varphi}_{v}(t)\right)=1,
\end{array} \\
\quad \begin{array}{r}
a_{2}^{1}\left(t, \bar{\varphi}_{u}(t), \bar{\varphi}_{v}(t)\right)=\cos (t)+3, \\
f_{1}\left(t, x, u, v, \bar{\varphi}_{u}(t), \bar{\varphi}_{v}(t)\right)=u+v+ \\
+\frac{\psi^{\prime}(t)-u_{x x}(t, \alpha, \beta)-u_{z z}(t, \alpha, \beta)-\psi-v(t, \alpha, \beta)}{F_{1}(t, \alpha, \beta)} F_{1}(t, x, z),
\end{array}
\end{gathered}
$$




$$
f_{2}\left(t, x, u, v, \bar{\varphi}_{u}(t), \bar{\varphi}_{v}(t)\right)=u+v+F_{2}(t, x, z) .
$$

The constants $\widetilde{p}_{i}=\{2,1\}, p_{i}=\max \left\{\widetilde{p}_{i}, 2\right\}=2$.

We verify the conditions of the theorem.

Condition 1.

$$
\begin{aligned}
\sum_{i=1}^{2}\left(\left|a_{1}^{i}\left(t, \bar{\varphi}_{u}(t), \bar{\varphi}_{v}(t)\right)\right|\right. & +\left|a_{2}^{i}\left(t, \bar{\varphi}_{u}(t), \bar{\varphi}_{v}(t)\right)\right|+ \\
& \left.+\left|b_{1}^{i}\left(t, \bar{\varphi}_{u}(t), \bar{\varphi}_{v}(t)\right)\right|+\left|b_{2}^{i}\left(t, \bar{\varphi}_{u}(t), \bar{\varphi}_{v}(t)\right)\right|\right)=5+\cos (t)+3 \leqslant 9 .
\end{aligned}
$$

Thus, the condition is satisfied when $\gamma_{1}=0$.

Condition 2 is satisfied by the assumptions on the input data (25).

Condition 3.

$$
\begin{aligned}
& \sum_{\substack{s=\left(s_{1}, s_{2}\right), s_{i}=0,1, \ldots, 4, i=1,2}}\left(\left|D_{x}^{s} f_{1}\left(t, x, u, v, \bar{\varphi}_{u}(t), \bar{\varphi}_{v}(t)\right)\right|+\left|D_{x}^{s} f_{2}\left(t, x, u, v, \bar{\varphi}_{u}(t), \bar{\varphi}_{v}(t)\right)\right|\right)= \\
& \quad=\sum_{\substack{s=\left(s_{1}, s_{2}\right), s_{i}=0,1, \ldots, 4 \\
i=1,2}} \mid \frac{\partial^{|s|}}{\partial x^{s_{1}} \partial z^{s_{2}}}(2(u+v)+ \\
& \left.\quad+\frac{\psi^{\prime}(t)-u_{x x}(t, \alpha, \beta)-u_{z z}(t, \alpha, \beta)-\psi-v(t, \alpha, \beta)}{F_{1}(t, \alpha, \beta)} F_{1}(t, x, z)+F_{2}(t, x, z)\right) \mid \leqslant \\
& \leqslant \sum_{\substack{s=\left(s_{1}, s_{2}\right), s_{i}=0,1, \ldots, 4 \\
i=1,2}} C\left|\frac{\partial^{|s|}}{\partial x^{s_{1}} \partial z^{s_{2}}}\left(\left(1+u+v+u_{x x}(t, \alpha, \beta)+u_{z z}(t, \alpha, \beta)+v(t, \alpha, \beta)\right)\right)\right| \leqslant \\
& \leqslant P_{1}\left(S_{u, v}(t)\right) .
\end{aligned}
$$

Consequently, condition 3 is satisfied when $\gamma_{2}=1$. Hence, the classical solution $u(t, x, z)$, $v(t, x, z)$ to the auxiliary direct problem $(26),(27)$ exists, for example, in the class $Z_{x, z}^{2,2}([0, T])$.

The research for this paper was carried out in Siberian Federal University within the framework of the project "Multidimensional Complex Analysis and Differential Equations" funded by the grant of the Russian Federation Government to support scientific research under supervision of a leading scientist, no. 14.Y26.31.0006.

\section{References}

[1] I.V.Frolenkov, G.V.Romanenko, On the Solvability of Special Systems of One-Dimensional Loaded Parabolic Equations and Composite-Type Systems with Cauchy Data, Journal of Applied and Industrial Mathematics, 8(2014), no. 2, 196-207.

[2] Yu.Ya.Belov, Inverse Problems for Partial Differential Equations, Utrecht, VSP, 2002

[3] N.N.Yanenko, Fractional Steps for Solving Multidimensional Problems of Mathematical Physics, Nauka, Novosibirsk, 1967 (in Russian). 
[4] G.V.Romanenko, A Representation of Solution of the Identification Problem of the Coefficients at Second Order Operator in the Multi-Dimensional Parabolic Equations System, Journal of Siberian Federal University. Mathematics \& Physics, 7(2014), no. 1, 100-111.

[5] I.V.Frolenkov, Yu.Ya.Belov, On the existence of solutions for a class loaded two-dimensional parabolic equations with the Cauchy data, Non-classical equations of mathematical physics. Novosibirsk, Institut Matematiki, (2012), 262-279 (in Russian).

[6] I.V.Frolenkov, M.A.Darzhaa, On the existence of solution of some problems for nonlinear loaded parabolic equations with Cauchy data, Journal of Siberian Federal University. Mathematics \& Physics, 7(2014), no. 2, 173-185.

[7] Yu.Ya.Belov, K.V.Korshun, On solvability of the Cauchy problem for a loaded system, Journal of Siberian Federal University. Mathematics \& Physics, 7(2014), no. 2, 155-161.

\section{О разрешимости системы двух многомерных нагруженных параболических уравнений с данными Коши}

\section{Галина В. Романенко}

Игорь В. Фроленков

Институт математики и фундаментальной информатики

Сибирский федеральный университет Свободный, 79, Красноярск, 660041

Россия

Исследована многомерная система двух параболических нагруженных уравнений специалъного вида в случае данных Коши. Получены достаточные условия существования решения в классе гладких ограниченных функиий. Для доказательства используется метод расщепления на дифференциальном уровне (метод слабой аппроксимации).

Ключевые слова: обратная задача, прямая задача, нагруженное уравнение, параболическое уравнение, метод слабой аппроксимации, системы уравнений в частных производных, задача Коши. 